\title{
¿Es la participación política convencional un indicador del compromiso cívico de los jóvenes?*
}

Is conventional Political Participation An
Indicator of Youth Civic Engagement?

Recibido: 28 de diciembre de 2012 | Revisado: 6 de octubre de 2013 | Aceptado: 19 de diciembre de 2014

\author{
EDMUNDO VARELA ** \\ Pontificia Universidad Católica de Chile, Santiago, Chile \\ MARÍA LORETO MARTÍNEZ *** \\ Pontificia Universidad Católica de Chile, Santiago, Chile \\ PATRICIO CUMSILle **** \\ Pontificia Universidad Católica de Chile, Santiago, Chile
}

doi:10.11144/Javeriana.upsy14-2.eppc

Para citar este artículo: Varela, E., Martínez, M.L., \& Cumsille, P. (2015). iEs la participación política convencional un indicador del compromiso cívico de los jóvenes? Universitas Psychologica, 14(2), 715730. http://dx.doi.org.10.11144/Javeriana.upsy14-2. eppc

* Este estudio ha sido realizado en el marco de una investigación financiada por el Fondo Nacional de Desarrollo Científico y Tecnológico de Chile (FONDECYT N 1085231), dirigida por los investigadores M. Loreto Martínez, e-mail:mlmartig@ uc.cl, y Patricio Cumsille, e-mail: pcumsill@uc.cl.

*** Estudiante de doctorado en Psicología, Pontificia Universidad Católica de Chile, Santiago, Chile. Av. Vicuña Mackenna 4860, Comuna de Macul, Santiago, Chile. Teléfono: (56)223545883. Correo electrónico: epvarela@uc.cl La correspondencia relativa a este artículo debe ser enviada a este autor.

**** Profesor titular. Escuela de Psicología, Pontificia Universidad Católica de Chile, Santiago, Chile.

***** Profesor titular. Escuela de Psicología, Pontificia Universidad Católica de Chile, Santiago, Chile.

\section{RESUMEN}

Aunque el compromiso cívico es un concepto multidimensional, con frecuencia la baja participación de los jóvenes en política convencional se interpreta como un indicador de menor compromiso cívico. Este estudio cuestiona la centralidad de la participación política convencional como principal indicador del compromiso cívico, y evalúa si la mayor atención hacia la política convencional es un indicador del compromiso cívico en jóvenes. Los hallazgos de un análisis de regresión logística en una muestra de jóvenes chilenos $(\mathrm{N}=390)$ clasificados como: (a) políticos $(\mathrm{N}=279)$ y (b) apolíticos $(\mathrm{N}=111)$ indican que la participación política convencional predice la pertenencia al grupo político, y la participación local al apolítico. Los niveles de tolerancia, competencias cívicas, y participación política no convencional no resultaron predictores de la pertenencia a alguno de los dos grupos. Los hallazgos confirman que la participación política convencional no es indicador principal del compromiso cívico y señalan la importancia de avanzar en formular modelos multidimensionales del compromiso cívico más allá de la política convencional.

Palabras clave

compromiso cívico; jóvenes; política convencional; regresión logística

\section{A B S T R A C T}

Although civic engagement is a multidimensional concept, the low participation of youth in conventional politics is often interpreted as an indicator of low civic engagement. This study questions the centrality of conventional political participation as the main indicator of civic engagement, and assesses whether attention to conventional politics is an indicator of civic engagement among young people. Findings of a logistic regression analysis on a sample of young Chileans $(N=390)$ classified as: (a) political $(N=$ 279) and (b) apolitical $(N=111)$ indicated that conventional political participation predicts membership to the political and local participation to the apolitical group. Levels of tolerance, civic competencies, participation in nonconventional politics did not predict group membership. Findings indicate that conventional political participation is not the main indicator of civic engagement, and suggest the need to advance multidimensional models civic engagement beyond conventional politics.

Keywords

civic engagement; conventional politics; logistic regression; youth 
Los hallazgos de estudios en diversos países muestran que los jóvenes han disminuido su participación política convencional respecto a las generaciones anteriores (Flanagan \& Levine, 2010; Instituto Nacional de la Juventud [INJUV], 2004, 2009; Putnam, 1996; Smith, 2005), una de las dimensiones habitualmente consideradas en el estudio del compromiso cívico (Flanagan, Syvertsen, \& Stout, 2007; Jenkins, 2005; Putnam, 1996). Para Ehrlich (2000) el compromiso cívico consiste en trabajar para promover la calidad de vida de la comunidad mediante procesos políticos y no políticos e involucra el desarrollo de conocimientos (e.g., derechos), habilidades (e.g., comunicar), valores (e.g., responsabilidad), y la motivación (e.g., eficacia política) que permitan hacer esta diferencia.

El énfasis de algunas investigaciones en la dimensión comportamental (e.g., votar, contactar autoridades; e.g., Keeter, Zukin, Andolina, \& Jenkins, 2002; Pattie, Seyd, \& Whiteley, 2003; Putnam, 1996, 2000) ha limitado la comprensión del compromiso cívico (Zaff, Boyd, Li, Lerner, \& Lerner, 2010), pues este también refiere a capacidades y motivaciones cívicas (Amna, 2012; Crocetti, Jahromi, \& Meeus, 2012; Zaff, Hart, Flanagan, Youniss, \& Levine, 2010). Aunque se ha considerado el compromiso cívico como multidimensional (Torney-Purta, Amadeo, \& Andolina, 2010) pocos estudios han examinado la asociación entre los niveles de atención a la política convencional (e.g., votar) con los niveles en otras dimensiones del compromiso cívico (e.g., competencias cívicas) en medidas actitudinales.

Según Ames (2013) los jóvenes han sido calificados de apáticos o apolíticos por una falta de responsabilidad cívica inferida de una menor participación electoral o desconocimiento de los asuntos públicos (Ekman \& Amna, 2012). La participación política describe actividades directas o indirectas que realizan los ciudadanos para influir en las decisiones o en la elección de los gobernantes (Verba $\&$ Nie, 1972) e incluye formas convencionales (e.g., participación electoral, activismo partidario) y no convencionales (e.g., actividades de protesta, contacto con autoridades) (Teorell, Torcal, \& Montero, 2007). El menor interés de los jóvenes en la parti- cipación política convencional ha sido asociado con un menor compromiso cívico (Putnam, 1996, 2000). En búsqueda de una mayor comprensión de los procesos de transformación política en el mundo Ocampo (2011) y Rodríguez (2005) plantean que los jóvenes serían parte de nuevos movimientos sociales que están ocurriendo en ámbitos más alejados de la política tradicional (e.g., votar, participar en un partido político) o como nuevas formas de expresar un interés por los temas ciudadanos.

Según Ames (2013) la idea sobre jóvenes apolíticos o poco comprometidos con los problemas de la sociedad sería más bien imprecisa pues aunque estos pudieran estar decepcionados o desinformados del acontecer político podrían estar interesados en los problemas sociales que subyacen a este. Según Rodríguez (2005) la participación actual de los jóvenes difiere de aquella de los años sesenta que era más ideologizada, formalizada (e.g., juventudes políticas, movimientos estudiantiles) y motivada por objetivos de cambios sociales y políticos estructurales en la sociedad que la actual, más informal, más horizontal y con causas más relacionadas con la vida cotidiana (e.g., derechos sexuales y reproductivos, libertad de expresión). Desde una perspectiva más amplia, Ocampo (2011) plantea que la pérdida de centralidad del Estado en la vida social contemporánea y el desencanto y frustración de los jóvenes respecto de las instituciones políticas ha disminuido el interés de estos por los asuntos asociados con el Estado. Específicamente, Ocampo, Méndez y Pavajeau (2008) sugieren una pérdida del sentido y significado de lo público, que se manifiesta en prácticas de apatía y baja participación de los ciudadanos en los asuntos que los afectan, en prácticas de exclusión política de algunos sectores de la población, o en el uso de lo público en beneficio de intereses particulares favoreciendo fenómenos sociales de corrupción, impunidad y violencia.

Si en la actualidad los jóvenes no comparten las formas convencionales de participación política (Martínez, 2010; Sandoval \& Hatibovic, 2010), inferir niveles de compromiso cívico desde la participación política convencional sería una limitada forma de estimar el compromiso cívico de los jóvenes. Según Amna (2012) el compromiso cívico 
refiere a valores, creencias, actitudes, sentimientos, conocimientos, habilidades, y comportamientos asociados con situaciones fuera del ámbito familiar y los amigos, que pueden expresarse con actos en el ámbito público, de mercado, civil, personal, o político. Muy pocos estudios sin embargo, han evaluado si jóvenes que expresan diferente grado de interés o atención a la política convencional como un predictor de la participación política (Sabucedo, Durán, Alzate, \& Barreto, 2010; Verba, Schlozman, \& Brady, 1995) presentan diferencias en otros aspectos del compromiso cívico. Los hallazgos de Sabucedo et al. (2010) muestran que la identificación política de los jóvenes orientada a ideas de derecha a izquierda se asocia positivamente con el interés por participar en política (e.g., $\beta=$ $0.17 ; p \leq 0.05$ ), aunque no consideran jóvenes que carezcan de esta identificación o que se consideren a sí mismos como apolíticos (Núñez, Jenaro, Flores, \& Guzmán, 2014).

Si suponemos entonces que el compromiso cívico incluye comportamientos (e.g., votar), actitudes (e.g., atención cívica), conocimientos (e.g., procesos democráticos) y motivaciones (e.g., eficacia política) (O’Neill, 2007), es relevante evaluar si diferentes grados de atención a la política convencional se asocian con diferentes niveles de compromiso cívico definido multidimensionalmente a través de dimensiones como competencias cívicas, tolerancia, participación política y cívica. Si la evidencia sugiere cambios en los niveles de participación política convencional en los jóvenes, ise puede inferir que la menor participación se asocia con menores niveles en otras dimensiones cívicas? Si efectivamente existe una asociación entre la participación política convencional con otras dimensiones del compromiso cívico, ise referirán estas más bien a las dimensiones políticas?

Los resultados de muestras representativas en Chile (INJUV, 2009) indican que 86\% de los jóvenes no está interesado en votar en elecciones políticas, y que 95\% no participaría en una campaña política. En cambio, 55\% de los jóvenes declara interés por participar de alguna organización que defienda alguna causa social y $90 \%$ considera que las personas jóvenes son un aporte importante para la sociedad (INJUV, 2009). Las cifras anteriores sugieren que los jóvenes se interesan por los asuntos públicos a pesar de su menor disposición hacia la participación política convencional. En efecto, la participación en actividades no convencionales como el voluntariado han aumentado (Flanagan \& Levine, 2010) de $4.5 \%$ (INJUV, 2004) a $9.9 \%$ (INJUV, 2009).

Posibles explicaciones de la menor atención de los jóvenes a la política convencional son que ellos definirían sus estilos de vida cada vez más desde sus propias decisiones individuales (Programa de las Naciones Unidas para el Desarrollo [PNUD], 2009). Los jóvenes tendrían nuevas formas de vincularse con otros y con los asuntos públicos (Ocampo, 2011; Rodríguez, 2005). Los jóvenes serían activos en la discusión pública a través de internet (Shah, Cho, Eveland, \& Kwak, 2005; Wyatt, Katz, \& Kim, 2000), lo que haría más accesible y transparente el acceso a la comunidad (Thomlinson, 1999) al simplificar los vínculos interpersonales (Ng \& Detenber, 2005) y facilitar los diálogos cívicos deliberativos (Dahlberg, 2001). Para Schlegel (2000) los jóvenes forman parte de una cultura juvenil global en la cual internet (56\% de los jóvenes la usa todos o casi todos los días; INJUV, 2009) permite una tecno-sociabilidad (Holmes \& Rusell, 1999) con nuevas formas de agencia ciudadana, de demandas sociales y de articular lo colectivo con lo individual (PNUD, 2009). Por lo tanto, futuros estudios del compromiso cívico en jóvenes debieran incluir medidas de su atención, información y participación cívica mediante internet (redes sociales) (Mossberger, Tolbert, \& McNeal, 2008).

Si las nuevas formas de construir capital social mediante conexiones de internet con amigos, familiares, redes sociales y organizaciones (Wellman, Haase, Witte, \& Hampton, 2001) también incluye la colaboración directa hacia otros miembros de la comunidad (Adler \& Goggin, 2005; Pleyers, 2004), sería posible suponer que los jóvenes expresan su compromiso cívico más allá de la participación política convencional (Muxel, 1994, 2001). Evaluar este compromiso centrado en el individuo (Crocetti et al., 2012; Zaff et al., 2010) debiera incluir habilidades (e.g., competencias cívicas) y actitudes (e.g., 
disposición cívica) que se asocian con las decisiones de participar en la vida cívica (Levinson, 2010). Por ejemplo, para Flanagan y Faison (2001) la tolerancia es esencial en una identidad democrática y las competencias cívicas representan las habilidades necesarias para lograr las metas cívicas de un grupo de ciudadanos.

Los hallazgos de estudios previos (Flanagan et al., 2007) también sugieren que las medidas de compromiso cívico debieran incluir formas de activismo no convencional (Barnes \& Kasse, 1979), la participación en torno a temáticas concretas (e.g., derechos indígenas, cuidado del medio ambiente) (Krauskopf, 2000) y también los comportamientos y las actitudes cívicas con que los jóvenes se proponen influir en las decisiones sobre los asuntos públicos (Conway, 1990). Por ejemplo, Haste y Hogan (2006) plantean que la política no convencional ha pasado de tener un rol periférico a central en el funcionamiento de las democracias. Entonces, estimar el compromiso cívico de los jóvenes requiere evaluar comportamientos cívicos (e.g., protestar), habilidades cívicas (e.g., contactar autoridades), conexiones cívicas (e.g., pertenecer a clubes o asociaciones cívicas) y responsabilidades cívicas (e.g., cumplir las leyes) (Zaff, Hart et al., 2010).

\section{Cambio en los contextos de socialización y en las formas de hacerse adulto}

También es posible analizar los cambios en la forma en que los jóvenes expresan su compromiso cívico respecto a la generación de sus padres (Putnam, 1996) en el contexto de la transición hacia la vida adulta (Flanagan \& Levine, 2010). En relación a la generación de sus padres las generaciones actuales han sido socializadas con diferentes expectativas respecto a los procesos de construcción de la identidad (Arnett, 2000; Lapeyronnie, 2005). La mayor elasticidad en las transiciones a la adultez (Fussell \& Furstenberg, 2005) y las mayores oportunidades socioeconómicas han permitido a los grupos más educados (Galambos \& Martínez, 2007) o en los países más desarrollados una mayor heterogeneidad en los caminos que pueden elegir (Bendit, Hahn, \& Miranda, 2008; Coté \& Bynner, 2008) para ha- cerse adultos. En un contexto global los jóvenes se interesarían por temas más allá de sus realidades locales (Arnett, 2002) y sus valores también estarían influidos por la prevalencia del sistema de mercado (Silva \& Silva, 2010). Para Flanagan (2008) la socialización de los jóvenes en el contexto de los valores de los sistemas neoliberales se asociaría con actitudes más individualistas, privilegiando logros educativos para lidiar con mercados laborales más inestables.

En síntesis los cambios de contextos y valores de socialización habrían permeado a los jóvenes de una cultura global (Arnett, 2002) basada en el individualismo, la democracia, el libre mercado, los derechos individuales, la apertura al cambio y la tolerancia (Giddens, 2000), y también de un sistema político neoliberal que se habría consolidado a nivel mundial (Pharr \& Putnam, 2000). En este contexto, la tarea de comprometerse con un sistema de valores y causas cívicas que dependen de las características culturales de cada sociedad y que evoluciona de acuerdo con los cambios que se producen en las sociedades a lo largo de la historia (Martínez, 2010) y de la forma en que los jóvenes internalizan estos elementos socioculturales para actuar de acuerdo al contexto sociopolítico en que viven (Ménard, 2010), implicaría que evaluar niveles de participación en la vida cívica requiere también estudiar los aspectos motivacionales (e.g., habilidades, actitudes) que asocian con formas actuales de expresar su compromiso cívico.

\section{Motivaciones para el compromiso cívico en la construcción de identidades juveniles}

Desde una perspectiva generacional (Mannheim, 1952) las nuevas formas de expresar el compromiso cívico se asociarían al ambiente global en que los jóvenes construyen su identidad y transitan a los roles adultos (e.g., trabajo, matrimonio) (Flanagan \& Tucker, 1999). Flanagan (2008) plantea que la conciencia de alcance global a través del acceso democrático a información y la participación mediante internet habría creado en los jóvenes 
identidades políticas más fluidas, flexibles e inclusivas. Según Beck (2005) el participar de procesos globales que trascienden la soberanía nacional haría que las motivaciones de los jóvenes sean más de persona a mundo y menos de ciudadano a nación. Krauskopf (2000) plantea que los jóvenes tendrían una mirada menos ideológica que sus padres y que incluiría temáticas menos universalistas de cambio social. Los jóvenes sentirían que a través del esfuerzo y la fijación de objetivos pueden salir adelante al valorar oportunidades, derechos civiles, políticos, económico-sociales y culturales existentes (Martínez, Silva, Morandé, $\&$ Canales, 2010), y diferenciándose de posturas de la generación de sus padres (e.g., solicitar ayuda al gobierno para salir de sus problemas) (Flanagan \& Tucker 1999; Flanagan, Ingram, Gallay, \& Gallay 1997; Tucker, 1999).

Los hallazgos anteriores sugieren que estudiar el compromiso cívico debiera considerar aspectos del desarrollo individual y de los contextos de socialización cívica (Flanagan, Martínez, \& Cumsille, 2009), preguntas no resueltas aún. Por ejemplo, Flanagan y Levine (2010) cuestionan si las generaciones actuales tienen conexiones permanentes más débiles con la vida cívica que las generaciones anteriores o si se debe a una transición más larga hacia la adultez por un desplazamiento de los marcadores de la edad adulta (e.g., tardan más en casarse o terminar su educación). Evaluar el compromiso cívico a nivel individual requeriría evaluar dimensiones más permanentes del desarrollo (e.g., actitudes) y otras quizás más contextuales (e.g., formas o niveles de participación). Para TorneyPurta et al. (2010) el compromiso cívico de los jóvenes incluiría la participación (no limitado al voto), la comprensión política (no limitada a los conocimientos), las actitudes y disposiciones (no limitado a la tolerancia) y el contexto (no limitado a los curriculum).

Considerando los posibles cambios en valores y contextos de socialización descritos y el compromiso cívico como un concepto multidimensional (Amna, 2012; Bobek, Zaff, Li, \& Lerner, 2009; O’Neill, 2007; Torney-Purta et al., 2010; Zaff et al, 2010), este estudio supone que el nivel de atención a la política convencional es un limitado indicador de otras dimensiones del compromiso cívico de los jóvenes pues este incluye las habilidades (e.g., competencias cívicas) y creencias (e.g., sentido de justicia social) que influyen en la participación cívica o política (Crocetti et al., 2012; Levinson, 2010)

Para contrastar este postulado el estudio examina si jóvenes con diferente grado de atención a la política convencional, estimado mediante la identificación con ideas políticas tradicionales (e.g., izquierda, derecha) o la no identificación con estas ideas (e.g., apolítico), difieren también en otras disposiciones cívicas (e.g., hacia la participación política no convencional, tolerancia, hacia la participación política o cívica), competencias cívicas (e.g., contactar autoridades) y concepciones de ciudadanía (e.g., responsabilidad, participación, justicia social). Si efectivamente la menor atención en los jóvenes hacia la política convencional se asocia con menores niveles de compromiso cívico, se esperarían diferencias significativas en las actitudes cívicas, las competencias cívicas y también las concepciones ciudadanas declaradas por estos dos grupos de jóvenes.

Es decir, si la atención hacia la política convencional como predictor de la participación política es un limitado indicador del compromiso cívico en jóvenes, se espera (1) que el grado de identificación con la política convencional no se asocie con diferencias significativas en los niveles de tolerancia o competencias cívicas. Si el compromiso cívico también incluye la participación política no convencional y la colaboración directa con la comunidad, se espera (2) que el grado de identificación con la política convencional no se asocie con diferencias significativas en los niveles de participación política no convencional y de participación local. Si la atención hacia la política convencional efectivamente se asocia con la participación política convencional, se espera (3) que el mayor grado de identificación con la política convencional se asocie con niveles significativamente mayores en los niveles de participación política convencional. Adicionalmente, si existe un rango amplio de ideas sobre cómo concebir a un buen ciudadano -que se pueden configurar en 
tres formas de expresar la ciudadanía tales como (a) personalidad responsable, (b) participativos y (c) orientados a la justicia (Westheimer \& Kahne, 2004) - se espera (4) que el grado de identificación con la política convencional se asocie a diferentes concepciones de ciudadanía como formas alternativas de expresar el compromiso cívico.

\section{Método}

\section{Participantes}

La muestra fue intencionada y está integrada por jóvenes $\left(N=390,42 \%\right.$ hombres, $M_{\text {edad }}=20$ años, $D E=1.79)$ reclutados en cinco universidades chilenas. En base a sus respuestas a un ítem de identificación en el instrumento que evaluó sus diferentes ideas políticas los participantes fueron asignados a dos grupos alternativos (i.e., político o apolítico). La Tabla 1 muestra que $72 \%$ de los participantes fue asignado al grupo político. El 42\% del grupo político y el $9 \%$ del apolítico estaban inscritos en los registros electorales (el promedio nacional se estima en 21\%; INJUV, 2009). El 54\% de los participantes pertenece a dos universidades de Santiago de Chile.

\section{Procedimiento}

Se contactó a los establecimientos educacionales para que autorizaran a invitar a sus alumnos a participar voluntariamente en el estudio. Se explicaron los objetivos, las condiciones de participación y los participantes firmaron un consentimiento escrito. Se aplicó un cuestionario de autoreporte en las salas de clases por examinadores capacitados para este efecto.

\section{Instrumento}

El instrumento utilizado evalúo las siguientes dimensiones:

\section{Identificación con ideas políticas}

Esta dimensión fue evaluada mediante un ítem que permitió asignar a cada participante al grupo político o al apolítico (e.g., considerando tus ideas políticas, ite sientes más cercano a?: Izquierda, Centro izquierda, Centro, Centro derecha, Derecha, Apolítico). El grupo político incluye a quienes declaran cercanía con alguna idea política (e.g., Izquierda, Derecha) y el apolítico a quienes se declaran apolíticos.

\section{TABLA 1}

Descripción de los Grupos Político y Apolítico, y Muestra total

\begin{tabular}{lccc}
\hline \multicolumn{1}{c}{ Medida } & Político & Apolítico & Total \\
\hline$N$ & 279 & 111 & 390 \\
Medad & $20(D E=1.87)$ & $19.9(D E=1.56)$ & $20(D E=1.79)$ \\
Hombres & $40 \%$ & $48 \%$ & $42 \%$ \\
ler año de Universidad & $60 \%$ & $65 \%$ & $62 \%$ \\
2do año de Universidad o más & $40 \%$ & $35 \%$ & $38 \%$ \\
Inscritos en registos electorales & $42 \%$ & $9 \%$ & $33 \%$ \\
Trabajo remunerado & $16 \%$ & $17 \%$ & $16 \%$ \\
Estudia en Santiago (CL/Chile) & $49 \%$ & $64 \%$ & $53 \%$ \\
NSE familiar: & & & \\
Alto & $14 \%$ & $7 \%$ & $60 \%$ \\
Medio alto & $59 \%$ & $61 \%$ & $28 \%$ \\
Medio bajo & $27 \%$ & $32 \%$ & \\
\hline
\end{tabular}

Nota. El nivel socioeconómico [NSE] familiar fue estimado mediante el nivel de educación de los padres (e.g., educación universitaria completa). Hasta el año 2011 para votar en Chile todo ciudadano que cumplía 18 años de edad debía inscribirse en los registros electorales, desde el año 2012 todo ciudadano que cumple 18 años quedan inscrito automáticamente en los registros electorales.

Fuente: elaboración propia 


\section{Tolerancia}

Esta escala evalúa el grado en que las personas aceptan puntos de vista distintos de los propios o aquellos que consideran como ciertos (Teven, McCroskey, \& Richmond, 1998). Está conformada por cuatro ítems (e.g., Me gusta hablar con gente que tiene puntos de vista diferentes a los míos). Las opciones de respuesta van desde 1 (Muy en desacuerdo) a 5 (Muy de acuerdo) y registra un índice de confiabilidad alfa de Cronbach de 0.76.

\section{Competencias cívicas}

Esta escala evalúa la disposición al cambio social, el activismo hacia la justicia social, los roles sociales y el empoderamiento político (Watts \& Flanagan, 2007). Está conformada por 8 ítems (e.g., Hacer un plan para atacar el problema). Las opciones de respuesta van desde 1 (Improbable) a 4 (Muy probable) y registra un índice de confiabilidad alfa de Cronbach de 0.83 .

\section{Actitudes sociopolíticas}

Estas dimensiones evalúan la disposición hacia tres formas generales de participación cívica como son: (a) participación política que representa las actividades para elegir a los líderes del gobierno; (b) voz política (i.e., participación política no convencional) que representa las actividades con que los individuos expresan sus opiniones políticas; y (c) participación cívica que representa acciones voluntarias organizadas para resolver problemas o ayudar a los demás (Jenkins, Andolina, Keeter, $\&$ Zukin, 2003). La escala que estudia estas tres dimensiones fue validada en la muestra de los 390 jóvenes mediante un análisis factorial exploratorio con método de extracción de ejes principales y rotación oblicua, de acuerdo al criterio de autovalores mayores a uno y las cargas factoriales de la matriz de estructura. Esta escala responde a una pregunta sobre la disposición a realizar ciertas actividades cívicas (e.g., Piensa en los próximos dos años de tu vida icuán probable es que hagas cada una de las siguientes actividades?). Las opciones de respuesta van desde 1 (Muy improbable) a 5 (Muy probable). Estas dimensiones están representadas por tres escalas:

\section{Participación política convencional.}

Esta escala evalúa la orientación hacia los procesos políticos (Jenkins, 2005). Está conformada por 3 ítemes (e.g., votar regularmente) y registra un índice de confiabilidad alfa de Cronbach de 0.74.

\section{Participación política no convencional.}

Esta escala evalúa la orientación hacia formas alternativas de compromiso cívico (Flanagan et al., 2007). Está conformada por 4 ítemes (e.g., unirse a una causa en contra de una compañía o una gran empresa) y registra un índice de confiabilidad alfa de Cronbach de 0.83 .

\section{Participación local.}

Esta escala evalúa la orientación a colaborar con la comunidad y ayudar a otros individuos (Haste \& Hogan, 2006). Está conformada por 3 ítemes (e.g., trabajar con un grupo para resolver un problema en la comunidad donde vives), y registra un índice de confiabilidad alfa de Cronbach de 0.80 .

\section{Concepciones de ciudadanía.}

Esta dimensión evalúa las diferentes formas de expresión del comportamiento de un buen ciudadano en una sociedad democrática (Westheimer, 2004). Está dimensión fue evaluada mediante una escala que responde a una pregunta sobre la definición como ciudadano (e.g., De las características que se describen a continuación, icuáles te definen a ti como ciudadano?) y los participantes deben elegir los tres ítems que mejor representen su concepto de ciudadanía. La escala se compone de 12 ítems que evalúan tres formas de expresar la ciudadanía: 6 ítems que evalúan ciudadanía responsable (e.g., sigo las reglas y las leyes), 4 ítems que evalúan ciudadanía participativa (e.g., expreso mi opinión acerca de asuntos sociales o políticos) y 2 ítems que evalúan 
TABLA 2

Regresión de Tolerancia, Competencias cívicas y Actitudes sociopolíticas

\begin{tabular}{lccccc}
\hline & & & & \multicolumn{2}{c}{ I.C. 95.0\% para OR } \\
\cline { 5 - 6 } \multicolumn{1}{c}{ Medida } & OR & Wald & $\mathrm{p}<$ & Inferior & Superior \\
\hline Edad & 0.947 & 0.396 & 0.529 & 0.800 & 1.122 \\
Género & 1.341 & 0.939 & 0.332 & 0.741 & 2.427 \\
NSE & 1.194 & 4.287 & 0.038 & 1.010 & 1.412 \\
Tolerancia & 0.980 & 0.008 & 0.930 & 0.620 & 1.547 \\
Competencias cívicas & 0.927 & 0.071 & 0.790 & 0.528 & 1.626 \\
Convencional & 2.992 & 31.413 & 0.000 & 2.039 & 4.389 \\
No convencional & 1.072 & 0.207 & 0.649 & 0.793 & 1.450 \\
Local & 0.673 & 4.874 & 0.027 & 0.473 & 0.957 \\
Constante & 0.446 & 0.129 & 0.720 & & \\
\hline
\end{tabular}

$\mathrm{VD}=1$ (i.e., grupo político), 0 (i.e., grupo apolítico)

Fuente: elaboración propia

ciudadanía orientada a la justicia (e.g., trabajo con otros para cambiar leyes injustas) (Westheimer \& Kahne, 2004).

\section{Plan de análisis}

Para contrastar las hipótesis de tolerancia, competencias cívicas, y actitudes sociopolíticas entre los grupos político y apolítico se efectuó un análisis de regresión logística. Para contrastar la hipótesis de si los grupos político y apolítico poseen formas alternativas de expresar su ciudadanía se efectuó un análisis de frecuencia y un análisis de varianza.

\section{Resultados}

Tolerancia, competencias cívicas, y actitudes sociopolíticas

En la Tabla 2 se presentan los resultados de la regresión logística que compara los grupos político y apolítico en las tres dimensiones evaluadas, tolerancia, competencias cívicas y actitudes sociopolíticas.

Las variables se ingresaron en dos bloques. Primero, las variables sociodemográficas (i.e., edad, género, nivel socioeconómico [NSE]) y segundo las dimensiones de tolerancia, competencias cívicas y actitudes sociopolíticas (i.e., convencional, no convencional, local). Se categorizó la variable de- pendiente con el valor 1 para el grupo político clasificado correctamente en un $98 \%$ por la regresión y con el valor 0 para el grupo apolítico clasificado correctamente en un 38\%. Los resultados indican que el activismo político convencional $(\mathrm{OR}=$ 2.992; $p=0.000$ ) es un predictor del grupo político y la participación local $(O R=0.637 ; p=0.027)$ es un predictor del grupo apolítico. Tal como muestra la Tabla 2 los resultados apoyan las hipótesis sobre tolerancia y competencias cívicas. Los resultados también apoyan las hipótesis de participación política convencional y no convencional, y no apoyan la de participación local.

Tal como se puede observar en la Tabla 1 la pregunta sobre identificación política permitió distinguir dos grupos de participantes. El $42 \%$ de los participantes que se identifican con alguna idea política se encuentra inscrito para votar versus el 9\% del grupo apolítico. Este resultado es consistente con los presentados en la Tabla 3 sobre promedios significativamente más altos del grupo político en actividades de participación formal como son un partido político tradicional, centro de alumnos o federación de estudiantes y algún colectivo que defiende una causa ideal. En la Tabla 3 se presentan distintas actividades de los jóvenes que responden a un enunciado común, "A continuación, se presenta una lista de organizaciones. Marca en cuáles participas regularmente o participaste anteriormente, 
TABLA 3

Anova de los grupos sobre frecuencia de participación en actividades sociales, cívicas, y políticas

\begin{tabular}{|c|c|c|c|c|}
\hline Participación & $\mathrm{MA}(\mathrm{DE})$ & $\mathrm{MP}(\mathrm{DE})$ & $\mathrm{F}$ & Sig. \\
\hline Club o grupo deportivo & $2.47(1.06)$ & $2.55(0.98)$ & 0.502 & 0.479 \\
\hline Grupo religioso o que cultiva la espiritualidad & $2.97(1.12)$ & $2.77(1.06)$ & 2.861 & 0.092 \\
\hline Centro de alumnos(as) o federación de estudiantes & $3.53(0.70)$ & $3.10(0.89)$ & 20.943 & 0.000 \\
\hline $\begin{array}{l}\text { Agrupación de ayuda a los demás (voluntariado, colonias } \\
\text { escolares, etc.) }\end{array}$ & $2.90(0.80)$ & $2.79(0.77)$ & 1.463 & 0.227 \\
\hline Partido político tradicional (juventudes políticas, etc.) & $3.92(0.31)$ & $3.54(0.75)$ & 26.242 & 0.000 \\
\hline Movimiento Guías y Scouts & $3.39(0.78)$ & $3.29(0.87)$ & 1.024 & 0.312 \\
\hline $\begin{array}{l}\text { Agrupación o grupo de hobbie o juego (ajedrez, cartas, } \\
\text { poker ) }\end{array}$ & $3.23(0.90)$ & $3.34(0.89)$ & 1.135 & 0.287 \\
\hline $\begin{array}{l}\text { Organización o agrupación artística y/o cultural (grupo de } \\
\text { teatro, grupo de música, banda, grupo de danza, etc.). }\end{array}$ & $2.75(0.84)$ & $2.67(0.84)$ & 0.632 & 0.427 \\
\hline $\begin{array}{l}\text { Comunidad o grupo virtual (grupo de chat, foros, blogs, } \\
\text { etc.) }\end{array}$ & $2.67(1.22)$ & $2.67(1.34)$ & 0.001 & 0.974 \\
\hline Sindicato u organización laboral o profesional & $3.56(0.55)$ & $3.43(0.66)$ & 3.196 & 0.075 \\
\hline Barra de fútbol & $3.32(1.04)$ & $3.45(0.94)$ & 1.365 & 0.243 \\
\hline Organización vecinal u organización comunitaria & $3.65(0.61)$ & $3.58(0.63)$ & 0.997 & 0.319 \\
\hline $\begin{array}{l}\text { Colectivo, agrupación o movimiento que defiende una } \\
\text { causa o ideal (ecológica, derechos humanos, derechos de } \\
\text { la mujer, derechos de las minorías sexuales, etc.) }\end{array}$ & $3.24(0.62)$ & $3.05(0.79)$ & 4.889 & 0.028 \\
\hline $\begin{array}{l}\text { Organización, agrupación o movimiento que lucha por un } \\
\text { problema concreto (pase escolar, gratuidad PSU, etc.) }\end{array}$ & $2.96(0.78)$ & $2.89(0.86)$ & 0.586 & 0.444 \\
\hline $\begin{array}{l}\text { Agrupación o tribu que cultiva una identidad (tecno, } \\
\text { pokemon, skinhead, gótico, etc.) }\end{array}$ & $3.78(0.64)$ & $3.74(0.74)$ & 0.338 & 0.561 \\
\hline
\end{tabular}

Nota. Un menor valor en la dimensión representa mayor nivel de participación. MA = Media apolítico, MP = Media político

Fuente: elaboración propia

en cuáles te gustaría participar y en cuáles no te interesa participar". Las alternativas de respuesta iban desde $1=$ participo activamente, hasta $4=$ no me interesa participar. Es decir, un menor número en la escala representa mayor participación.

En la Tabla 3 se puede observar también que los jóvenes del grupo apolítico no presentan diferencias significativas respecto al grupo político en cuanto a su participación en una agrupación de ayuda a los demás, sindicato u organización comunitaria, organización vecinal u organizaciones comunitarias, organización o agrupación que luche por un problema en común.

En síntesis los jóvenes con y sin identificación con la política convencional representan a dos grupos que difieren en su interés por un activismo más político y que muestran niveles similares en tolerancia, competencias cívicas y participación política no convencional, difieren en participación política convencional y participación local.

\section{Concepciones de ciudadanía}

En la Figura 1 se presentan los resultados del análisis de varianza para contrastar si los grupos clasificados como político y apolítico difieren en sus concepciones de ciudadanía.

Los resultados del análisis de frecuencia (i.e., porcentaje de preferencia de cada ítem) y de varianza para los doce ítems de ciudadanía evaluados indican que en su concepción de ciudadanía el grupo político prioriza la responsabilidad formal (i.e., votar regularmente; $p=$ 0.002) y la participación política (i.e., expresar la opinión acerca de asuntos sociales y políticos; $p=0.004)$. Aunque no existen diferencias significativas entre el grupo político y apolítico en otros indicadores 
de sus concepciones de ciudadanía, un análisis de varianza considerando género indican que en sus concepciones de ciudadanía, los hombres del grupo apolítico priorizan la responsabilidad hacia otras personas (i.e., ayudar a quienes lo necesitan, ser considerado con los demás, $p=0.037$ ).

\section{Discusión}

Tal como se hipotetizó, los resultados de este estudio indican una limitada asociación entre la atención hacia la política convencional y otras dimensiones del compromiso cívico. Los resultados también indican que los jóvenes con y sin identificación con la política convencional tienen niveles similares en otras dimensiones del compromiso cívico como las competencias cívicas o la tolerancia y formas alternativas de expresar su ciudadanía. Estos hallazgos son consistentes con la frecuencia que los jóvenes manifiestan involucrarse en actividades cívicas como una organización vecinal u organización comunitaria o una agrupación de ayuda a los demás en grado similar entre ambos grupos y una mayor participación en centros de alumnos o un partido político en el grupo político (e.g., Tabla 3). Si el grupo político declara niveles significativamente superiores en su disposición a la participación política convencional y el grupo apolítico declara niveles significativamente superiores en su disposición a la participación local y no existen diferencias significativas en los otros indicadores considerados para estudiar el compromiso cívico (i.e., competencias cívicas, tolerancia, participación política no convencional), es posible concluir que existen diferentes formas de expresar compromiso cívico o que no se limitan a la participación política convencional.

Aunque la hipótesis de la participación local postulaba similitudes entre los grupos, la mayor disposición del grupo apolítico a colaborar con su comunidad inmediata es un indicador de compromiso cívico en jóvenes que se declaran indiferentes hacia la política convencional. Este hallazgo evidencia la necesidad de evaluar el compromiso cívico de forma multidimensional, pues los jóvenes del grupo apolítico habrían sido calificados como

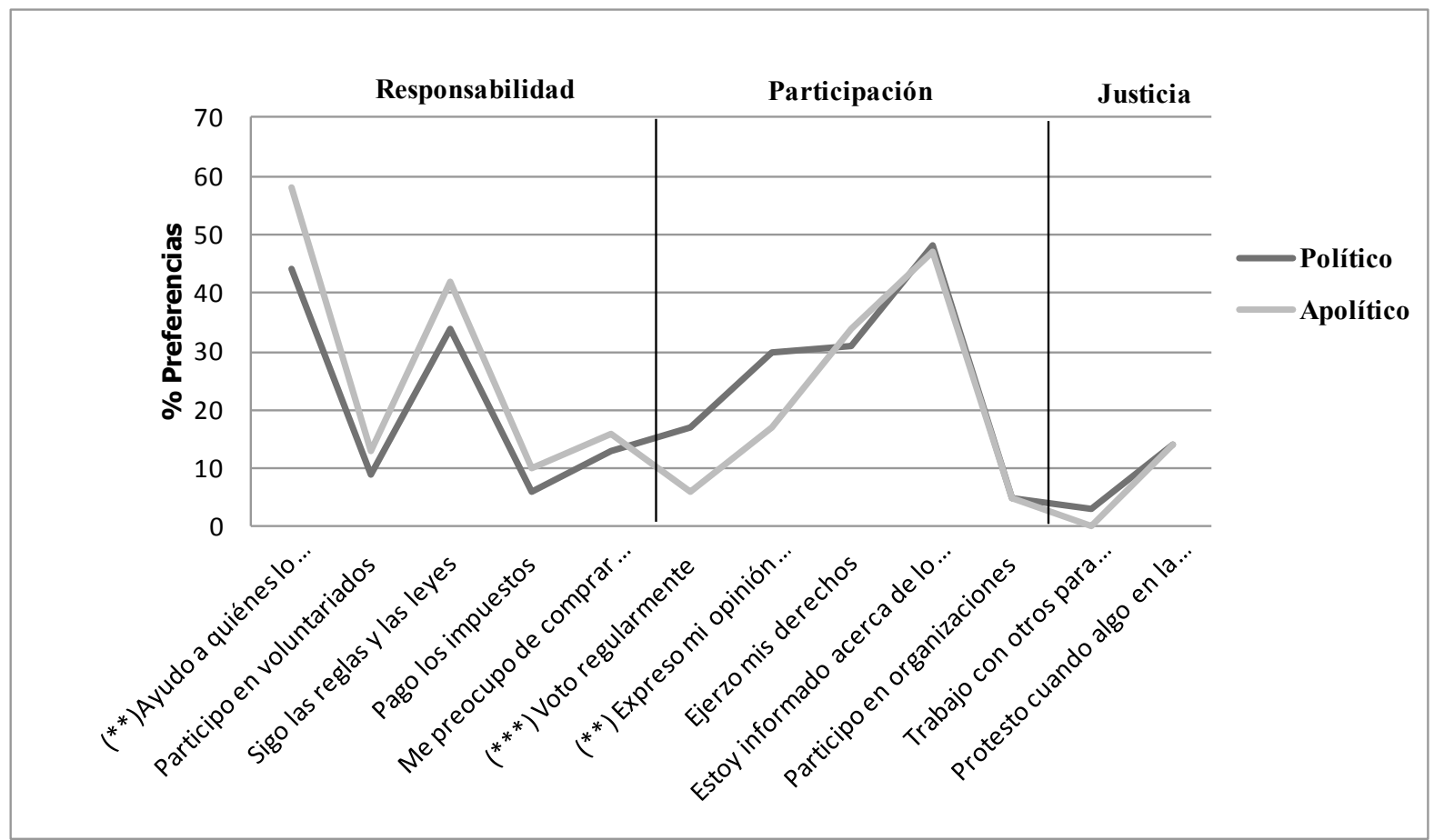

Figura 1. Concepciones de ciudadanía. El grupo político prioriza la participación formal. El grupo apolítico priorizaría la responsabilidad. Adaptado de "What Kind of Citizen: The Politics of Educating for Democracy" de J. Westheimer E J. Kahne, 2004. American Educational Research Journal, 41 (2), 237-269. 
poco comprometidos si solo se considera su grado de atención hacia la política convencional o su participación política convencional para estimar su grado de compromiso cívico. Si recordamos la definición seminal de Putnam (1996) "el compromiso cívico es la conexión de las personas con su comunidad y no sólo en los aspectos políticos" (p. 3). Esta mayor disposición del grupo apolítico hacia la participación local confirma que el compromiso cívico es más amplio que la participación política (Ménard, 2010; Tabla 3). Si el grupo apolítico muestra niveles similares de disposición hacia la participación política no convencional con el grupo político esto representa otro indicador de compromiso cívico en jóvenes indiferentes hacia la política convencional. Haste y Hogan (2006) plantean que el compromiso cívico se asocia con el mantenimiento de la democracia y quienes más apoyan la democracia son también los más dispuestos a protestar contra la autoridad y cuestionarla (Passini \& Morselli, 2011).

Además de relevar la necesidad de estudiar el compromiso cívico de los jóvenes de forma multidimensional, los hallazgos de estudio evidencian la necesidad de especificar las dimensiones que lo integran (Bobek et al., 2009; O`Neill, 2007; Zaff et al., 2010) y de especificar el objeto de estudio del compromiso cívico (Sherrod, Torney-Purta, \& Flanagan, 2010; Wilkenfeld, Lauckhardt, \& TorneyPurta, 2010). Según los hallazgos de este estudio además de la participación política convencional sería necesario incluir medidas de habilidades (e.g., competencias cívicas), actitudes (e.g., tolerancia) y otras medidas comportamentales (e.g., participación política no convencional, participación local) para estimar el compromiso cívico.

Los resultados del análisis de las concepciones de ciudadanía indican que el grupo político concibe su ciudadanía como participación formal. El grupo político manifiesta niveles significativamente superiores tanto en su disposición a votar como en expresar su opinión sobre asuntos sociales o políticos. Alternativamente, el grupo apolítico concibe la ciudadanía como responsabilidad hacia su comunidad más inmediata. Aunque este estudio no consideraba un análisis de género los resultados muestran que los hombres del grupo apolítico presentan niveles significativamente superiores en su disposición a ayudar a otros. Estos resultados sugieren que los dos grupos tienen formas propias de concebir la ciudadanía (Ocampo, Méndez, \& Pavajeau, 2008) y que resultan consistentes con estudios anteriores en poblaciones diferentes. Por ejemplo, Haste y Hogan (2006) encontraron que las concepciones de ciudadanía de los jóvenes incluye el cumplimiento de la ley (e.g., votar) como un deber importante, pero colaborar con la comunidad (e.g., ayudar a otros) lo clasifican como igual o más importante que votar y mucho más importante que pertenecer a un partido político (Pattie, Seyd, \& Whiteley, 2004).

La validez de los hallazgos de este estudio debe interpretarse considerando algunas limitaciones de ajuste de la regresión logística y del formato de respuesta de las concepciones de ciudadanía. La inferencia de mayor disposición a la participación local del grupo apolítico debe interpretarse con cautela, pues la regresión predice en nivel medio-bajo al grupo apolítico. El formato de frecuencia de las respuestas de los participantes utilizado para estudiar sus concepciones de ciudadanía presenta limitaciones pues cada participante pondera tres ítems de los doce que posee la escala, futuros estudios debieran considerar el uso de una escala tipo Likert.

Aunque la participación política convencional es fundamental en la construcción del sistema democrático, los hallazgos sugieren reevaluar su centralidad como indicador de compromiso cívico en jóvenes. La participación política sería una forma más propia de la participación adulta $\left(\mathrm{O}^{\prime} \mathrm{Neill}\right.$, 2007; Ocampo, 2011; Rodríguez, 2005) que se evidencia en la correlación positiva entre la edad y la participación en política convencional (Norris, 2003; O’Neill, 2007). La limitada capacidad de la política convencional como indicador de compromiso cívico en jóvenes debiera motivar investigaciones tanto sobre los predictores específicos de la participación cívica o política (Sabucedo et al., 2010) de los jóvenes como sobre los indicadores que mejor representan el compromiso cívico (Zaff et al., 2010). El grado de consistencia entre los resultados de las cuatro hipótesis sugiere que futuros estudios estimen qué tipo de relación existe entre el compromiso cívico y formas alternativas de concebir 
la ciudadanía. Esto colaboraría con la necesidad del desarrollo de un marco teórico en compromiso cívico (Wilkenfeld et al., 2010), pues diferentes concepciones de ciudadanía llevan a distintas creencias respecto a las capacidades y las formas de comprometerse que poseen los ciudadanos (Westheimer $\&$ Kahne, 2004).

\section{Referencias}

Adler, R. P. \& Goggin, J. (2005). What Do We mean By "Civic Engagement'?" Journal of Transformative Education, 3(3), 236-253.

Ames, M. (2013). Engaging "Apolitical" Adolescents: Analyzing the Popularity and Educational Potential of Dystopian Literature Post-9/11. The High School Journal, 97(1), 3-20.

Amna, E. (2012). How is civic engagement developed over time? Emerging answers from a multidisciplinary field. Journal of Adolescence, 35(3), 611-627.

Arnett, J. J. (2000). Emerging adulthood: A theory of development from the late teens through the twenties. American Psychologist, 55(5), 469-480.

Arnett, J. J. (2002). The Psychology of Globalization. American Psychologist, 57(10), 774-783.Barnes, S., \& Kaase, M. (Eds.) (1979). Political Action: Mass participation in five western societies. California: Sage.

Beck, U. (2005). Power in the global age. Cambridge, UK, Polity.

Bendit, R., Hahn, M., \& Miranda, A. (2008). Los jóvenes y el futuro. Procesos de inclusión social y patrones de vulnerabilidad en un mundo globalizado. Buenos Aires: Prometeo.

Bobek, D., Zaff, J., Li, Y., \& Lerner, R. (2009). Cognitive, emotional, and behavioral components of civic action: Towards an integrated measure of civic engagement. Journal of Applied Developmental Psychology, 30, 615-627.

Conway, M. M. (1990). Political participation in the United States. New York: Quarterly Press.

Coté, J. E., \& Bynner, J. M. (2008). Changes in the transition to adulthood in the UK and Canada: the role of structure and agency in emerging adulthood. Journal of Youth Studies, 11(3), 251-268.
Crocetti, E., Jahromi, P., \& Meeus, W. (2012). Identity and civic engagement in adolescence. Journal of Adolescence, 35, 521-532.

Dahlberg, L. (2001). Computer-mediated communication and the public sphere: A critical analysis. Journal of Computer-Mediated Communication, 7(1).

Ehrlich, T. (Ed.). (2000). Civic responsibility and higher education. Phoenix, AZ: American Council on Education/Oryx Press.

Ekman, J., \& Amna, E. (2012). Political participation and civic engagement: Towards a new typology. Human affairs, 22(3), 283-300.

Flanagan, C. A. (2008). Private anxieties and public hopes: The perils and promise of youth in the context of globalization. In J. Cole and D. Durham, (Eds.), Figuring the future: Children, youth, and globalization (pp. 125-150). Santa Fe, New Mexico: SAR Press.

Flanagan, C. A., \& Faison, N. (2001). Youth civic development: implications of research for social policy and programs. In L. Sherrod (Ed.) Social Policy Report: A Publication for the Society for Research in Child Development (pp. 3-14). Ann Arbor, MI: Soc. Res. Child Dev.

Flanagan, C. A., Ingram, P., Gallay, E. M.,\& Gallay, E. E. (1997). Why are people poor? Social conditions and adolescents' interpretation of the "social contract.” In R. D. Taylor, \& M. C. Wang (Eds.), Social and emotional adjustment and family relations in ethnic minority families (pp. 53-62). Mahwah, NJ: Erlbaum.

Flanagan, C. A., \& Levine, P. (2010). Civic Engagement and the Transition to Adulthood. Future of Children, 20(1), 159-179.

Flanagan, C. A, Martínez, M. L., \& Cumsille, P. (2009). Civil societies as cultural and developmental contexts for civic identity formation. In L. Arnett Jensen (Ed.), Bridging cultural and developmental psychology: New syntheses in theory, research and policy (pp. 113 - 37). New York: Oxford University Press.

Flanagan, C. A., Syvertsen, A. K., \& Stout, M. D. (2007). Civic measurement models: Tapping adolescents' civic engagement (CIRCLE Working Paper, 55). College Park, MD: Center for Information and Research on Civic Learning and Engagement. 
Flanagan, C. A., \& Tucker, C. J. (1999). Adolescents'explanations for political issues: Concordance with their views of self and Society. Developmental Psychology, 35(5), 1198-1209.

Fussell, E., \& Furstenberg, F.F. Jr. (2005). The Transition to Adulthood during the Twentieth Century: Race, Nativity, and Gender. In R. A. Settersten Jr., F. F. Furstenberg Jr., \& R. Rumbaut (Eds.), On the Frontier of Adulthood: Theory, Research, and Public Policy (pp. 29 -75). Chicago, IL: The University of Chicago Press.

Galambos, N. L., \& Martínez, M. L. (2007). Poised for emerging adulthood in Latin America: A pleasure for the privileged. Child Development Perspectives, 1(2), 109-114.

Giddens, A. (2000). Runaway world: How globalization is reshaping our lives. New York: Routledge.

Haste, H., \& Hogan, A. (2006). Beyond conventional civic participation, beyond the moral political divide: Young people and contemporary debates about citizenship. Journal of Moral Education, 35(4), $473-493$

Holmes, D., \& Russell, G. (1999). Adolescent CIT use: Paradigm shifts for educational and cultural practices? British Journal of Sociology of Education, 20(1), 69-78.

Instituto Nacional de la Juventud - INJUV (2004). Cuarta Encuesta Nacional de la Juventud. La integración social de los jóvenes en Chile 1994-2003. Santiago, Chile: Ministerio de Planificación y Cooperación.

Instituto Nacional de la Juventud - INJUV (2009). Sexta Encuesta Nacional de la Juventud. Santiago, Chile: INJUV.

Jenkins, K. (2005). Gender and Civic Engagement: Secondary Analysis of Survey Data (CIRCLE Working paper 41). College Park, MD: The Center for Information and Research on Civic Learning and Engagement.

Jenkins, K., Andolina, M. W., Keeter, S., \& Zukin, C. (2003). Is Civic Behavior Political? Exploring the Multidimensional Nature of Political Participation. Paper presented at the annual conference of the Midwest Political Science Association, Chicago.

Keeter, S., Zukin, C., Adnolina, M. W. \& Jenkins, K. (2002). The civic and political health of the nation:
A generational portrait. College Park: Center for Information and Research on Civic Learning and Engagement (CIRCLE), University of Maryland.

Krauskopf, D. (2000). Dimensiones críticas en la participación social de las juventudes. In S. Balardini (Ed.). La participación social y política de los jóvenes en el horizonte del nuevo siglo (pp. 119- 134). Buenos Aires, Argentina: CLACSO.

Lapeyronnie, D. (2005). L'engagement à venir. In V. Becquet \& C. De Linares (Eds.). Quand les jeuness'engagent. Entre expérimentations et constructions identitaires (pp. 35-53). Paris: L'Harmattan.

Levinson, M. (2010). The civic empowerment gap: Defining the problem and locating solutions. In L. R. Sherrod, J. Torney-Purta and C. Flanagan (Eds.). Handbook of research on civic engagement in youth (pp. 331-361). Hoboken: Wiley and Sons.

Mannheim, K. (1952/1928). The problem of generations. In P. Kecshevich (Ed.), Essays on the sociology of knowledge (pp. 276-322). London: Routledge \& Kegan Paul.

Martínez, M. L. (2010). Sección Especial: Desarrollo Cívico. Psykhe, 19(2), 3-4.

Martínez, M. L., Silva, C. L., Morandé, M., \& Canales, L. (2010). Los jóvenes ciudadanos: reflexiones para una política de formación ciudadana juvenil. Ultima década, 32, 105-118.

Ménard, M. (2010). Youth civic engagement. Background paper. Library of Parliament, 23. Ottawa, Cánada.

Mossberger, K., Tolbert C. J., \& McNeal, R. S. (2008). Digital Citizenship: The Internet, Society, and Participation. Cambridge: MIT Press.

Muxel, A. (1994). Jeunes des années quatre-vingt-dix: à la recherche d'une politique sans etiquette. In $\mathrm{P}$. De Perrineau (Ed.). Engagement politique. Déclin ou mutation? (pp. 239-266). Paris: Presses de la Fondation Nationale des Sciences Politiques.

Muxel, A. (2001). L'expérience politique des jeunes. Paris: Presses de Sciences Politiques.

Ng, E., \& Detenber, B. (2005). The impact of synchronicity and civility in online political discussions on perceptions and intentions to participate. Journal of Computer-Mediated Communication, 10(3).

Norris, P. (2003). Young People and Political Activism: From the Politics of Loyalties to the Politics of 
Choice? Report for the Council of Europe Symposium, 11, 27-28.

Núñez, M. J. B., Jenaro, C., Flores, N., \& Guzmán, K. (2014). Factores culturales asociados a las conductas sexuales en estudiantes universitarios de Uruguay y España: Estudio Preliminar. Psicología, Conocimiento y Sociedad, 4(1), 6-32.

Ocampo, A. M. (2011). Ciudadanía juvenil, juventud y Estado: Discursos de gobierno sobre sus significados. Revista Latinoamericana de Ciencias Sociales, Niñez y Juventud, 9(1).

Ocampo, A. M., Méndez, S., \& Pavajeau, C. (2008). Las subjetividades como centro de la formación ciudadana. Universitas psychologica, 7(3), 837-851.

O'Neill, B. (2007). Indifferent or just different? The political and civic engagement of young people in Canada. Ottawa: Canadian Policy Research Network.

Passini, S., \& Morselli, D. (2011). In the Name of Democracy: Disobedience and Value-Oriented Citizenship. Journal of Community and Applied Social Psychology, 21(3), 255-267.

Pattie, C., Seyd, P., \& Whiteley, P. (2003). Citizenship and Civic Engagement: Attitudes and Behaviors in Britain. Political Studies, 51, 443-468.

Pattie, C., Seyd, P., \& Whiteley, P. (2004). Citizenship in Britain: values, participation and democracy. Cambridge: Cambridge University Press.

Pharr, S. J., \& Putnam, R. D. (Eds.) (2000). Disaffected Democracies: What's Troubling the Trilateral Countries? Princeton: Princeton University Press.

Pleyers, G. (2004). Des black blocks aux alters-activistes: pôles et formes d'engagement des jeunes altermondialistes. Lien Social et Politiques, 51, 123-134.

Programa de las Naciones Unidas para el Desarrollo PNUD. (2009). Informe sobre desarrollo humano para el Mercosur 2009-2010. Innovar para incluir: Jóvenes y desarrollo humano. Buenos Aires: Libros del Zorzal, PNUD.

Putnam, R. D. (1996). The strange disappearance of civic America. The American Prospect, 24, 34-48.

Putnam, R. D. (2000). Bowling Alone: The Collapse and Revival of American Community, New York: Simon and Schuster.

Rodríguez, E. (2005). Jóvenes, Movimientos Juveniles y Políticas Públicas de Juventud en la Región Andina: Heterogeneidad de Situaciones, Diversidad de Soluciones. Montevideo: CELAJU-BANCO MUNDIAL-UNESCO.

Sabucedo, J., Durán, M., Alzate, M., \& Barreto, I. (2010). Emotions, ideology and collective political action. Universitas Psychologica, 10, 27-34.

Sandoval, J., \& Hatibovic, F. (2010). Socialización política y juventud: el caso de las trayectorias ciudadanas de los estudiantes universitarios de la región de Valparaíso. Ultima Década, 18(32), 11-36.

Schlegel, A. (2000). The global spread of adolescent culture. In L. Crockett \& R. K. Sibereisen (Eds.) Negotiating adolescence in time of social change (pp. 71-78). Cambridge: Cambridge University Press.

Shah, D. V., Cho, J., Eveland, W. P., \& Kwak, N. (2005). Information and expression in a digital age: Modeling Internet effects on civic participation. Communication Research, 32, 531-565.

Sherrod, L., Torney-Purta, J., \& Flanagan, C., (Eds.) (2010). Introduction. Research on the development of citizenship: a field comes of age. Handbook of research on civic engagement in youth (pp.1-20). Hoboken, NJ: John Wiley \& Sons.

Silva, E., \& Silva, C. L. (2010). La economía política y las motivaciones de participación socio-política de jóvenes chilenos: Una interpretación de los hallazgos de Martínez, Silva y Hernández (2010). Psykhe, 19(2), 39-50.

Smith, T. (2005). Generation gaps in attitudes and values from the 1970's to the 1990's. In Settersten, R. A., Jr., Furstenberg, F. F., Jr., \& Rumbaut, R. G. (Eds.). (2005). On the Frontier of Adulthood: Theory, Research, and Public Policy. Chicago, IL: The University of Chicago Press.

Teorell, J., Torcal, M., \& Montero, J. R. (2007). Political Participation: Mapping the Terrain. In J. W. van Deth, J. R. Montero, \& A. Westholm (Eds.). Citizenship and Involvement in European Democracies: A Comparative Analysis. London and New York: Routledge.

Teven, J. J., Richmond, V. P., \& McCroskey, J. C. (1998). Measuring tolerance for disagreement. Communication Research Reports, 15, 209-217.

Thomlinson, J. (1999). Globalization and Culture. Chicago: University of Chicago Press.

Torney-Purta, J., Amadeo, J., \& Andolina, M. W. (2010). A conceptual framework and multimethod ap- 
proach for research on political socialization and civic engagement. In L. R. Sherrod, J. TorneyPurta, and C. A. Flanagan (Eds.), Handbook of research on civic engagement in youth (pp. 497-523). Hoboken, NJ: Wiley.

Tucker, C. M. (1999). African American children: A self-empowerment approach to modifying behavior problems and preventing academic failure. Needham Heights, MA: Allyn \& Bacon.

Verba, S., \& Nie, N. H. (1972). Participation in America. New York: Harper \& Row.

Verba, S., Schlozman, K. L., \& Brady, H. E. (1995). Voice and equality: Civic voluntarism in American politics (Vol. 4). Cambridge, MA: Harvard University Press.

Watts, R. J., \& Flanagan, C. A. (2007). Pushing the envelope on youth civic engagement: A developmental and liberation psychology perspective. Journal of Community Psychology, 35(6), 779-792.

Wellman, B., Haase, A. Q., Witte, J., \& Hampton, K. (2001). Does the Internet Increase, Decrease or Supplement Social Capital? Social Networks, Participation, and Community Commitment. American Behavioral Scientist, 45(3), 436-455.

Westheimer, J. (2004). The politics of civic education. PS: Political Science $\mathfrak{E}$ politics, 38(2), 57-62.
Westheimer, J., \& Kahne, J. (2004). What Kind of Citizen: The Politics of Educating for Democracy. American Educational Research Journal, 41(2), 237-269.

Wilkenfeld, B., Lauckhardt, J., \& Torney-Purta, J. (2010). The relation between developmental theory and measures of civic engagement research on adolescents. In L. Sherrod, J. Torney-Purta., $\&$ C. Flanagan (Eds.). Handbook of research on civic engagement in youth (pp. 193-219). Hoboken, NJ: Wiley.

Wyatt, R. O., Katz, E., \& Kim, J. (2000). Bridging the spheres: Political and personal conversation in public and private spaces. Journal of Communication, 50(1), 71-92.

Zaff, J. F., Boyd, M., Li, Y., Lerner J. V., \& Lerner, R. M. (2010). Active and Engaged Citizenship: Multigroup and Longitudinal Factorial Analysis of an Integrated Construct of Civic Engagement. Journal of Youth and Adolescence, 39(7), 736-750.

Zaff, J. F., Hart, D., Flanagan, C. A., Youniss, J., \& Levine, P. (2010). Developing civic engagement within a civic context. In A. M. Freund \& M. E. Lamb (Eds.). Social and emotional development across the life span: Vol. 2, Handbook of life-span development. (pp. 591-623). Hoboken, NJ: John Wiley \& Sons. 
